Musées, Patrimoine et Culture scientifiques et techniques

$153 \mid 2014$

mai-juin 2014

\title{
Bases de données informatisées, inventaire légal et inventaire documentaire
}

\section{Pierre Dalous}

\section{OpenEdition \\ Journals}

Édition électronique

URL : http://journals.openedition.org/ocim/1383

DOI : $10.4000 /$ ocim. 1383

ISSN : 2108-646X

Éditeur

OCIM

Édition imprimée

Date de publication : 25 juin 2014

ISSN : 0994-1908

Référence électronique

Pierre Dalous, «Bases de données informatisées, inventaire légal et inventaire documentaire », La Lettre de l'OCIM [En ligne], 153 | 2014, mis en ligne le 25 juin 2016, consulté le 01 mai 2019. URL: http://journals.openedition.org/ocim/1383 ; DOI : 10.4000/ocim.1383 


\section{Bases de données informatisées, inventaire légal et inventaire documentaire}

\section{Pierre Dalous}

Conservateur du patrimoine au muséum d'Histoire naturelle de Toulouse

La particularité des musées d'Histoire Naturelle est de conserver dans leurs fonds, de grandes quantités d'objets de nature différente. Ces collections sont généralement organisées par discipline. Jusqu’au début des années 1980, la gestion des collections reposait essentiellement sur les connaissances et la mémoire des personnels des établissements. C'est à ce moment que l'informatique fit son apparition dans les musées.

Au muséum d'Histoire Naturelle de Toulouse, cette arrivée s'est traduite par l'acquisition en 1986 du logiciel MODES (Museum Object Data Entry System). Sans être un logiciel de gestion, cette base de données a permis d'effectuer les premières saisies de notices et de s'interroger concrètement sur les grandes problématiques auxquelles l'établissement allait être confronté. Ainsi un an plus tard, sous l'égide de la Direction des Bibliothèques, des Musées et de l'Information Scientifique et Technique (DBMIST, ministère de l'Éducation nationale), furent mis au point les bordereaux de saisie minimums. Ces bordereaux reflètent bien la vision naturaliste des collections que nous avions à l'époque. Du statut administratif, il n'était nullement question! Nous étions encore loin des fameuses 18 colonnes nécessaires à l'établissement du registre d'inventaire réglementaire.

C'est seulement 10 ans plus tard, qu'eut lieu la migration vers un véritable logiciel de gestion des collections. Deux postes informatiques, chacun équipé d'une version mono poste de la base de données, furent acquis, l'un consacré aux sciences de la Vie et l'autre aux sciences de la Terre.

À l'occasion du chantier de rénovation du muséum, avec l'arrivée d'assistants de conservation en charge de la gestion des collections, les bases évoluèrent en version réseau. De plus, une base « Sciences humaines » fit son apparition, plus adaptée aux collections d'ethnographie et de préhistoire. Toutefois la priorité étant donnée à l'ouverture du nouvel établissement, l'activité autour de la base de données resta très faible.

Ce n'est qu'en 2008, après l'ouverture du muséum, que ce travail fut réellement engagé. Il a commencé par la formation des personnels. Celle-ci a notamment permis de lever le doute concernant le potentiel de nos

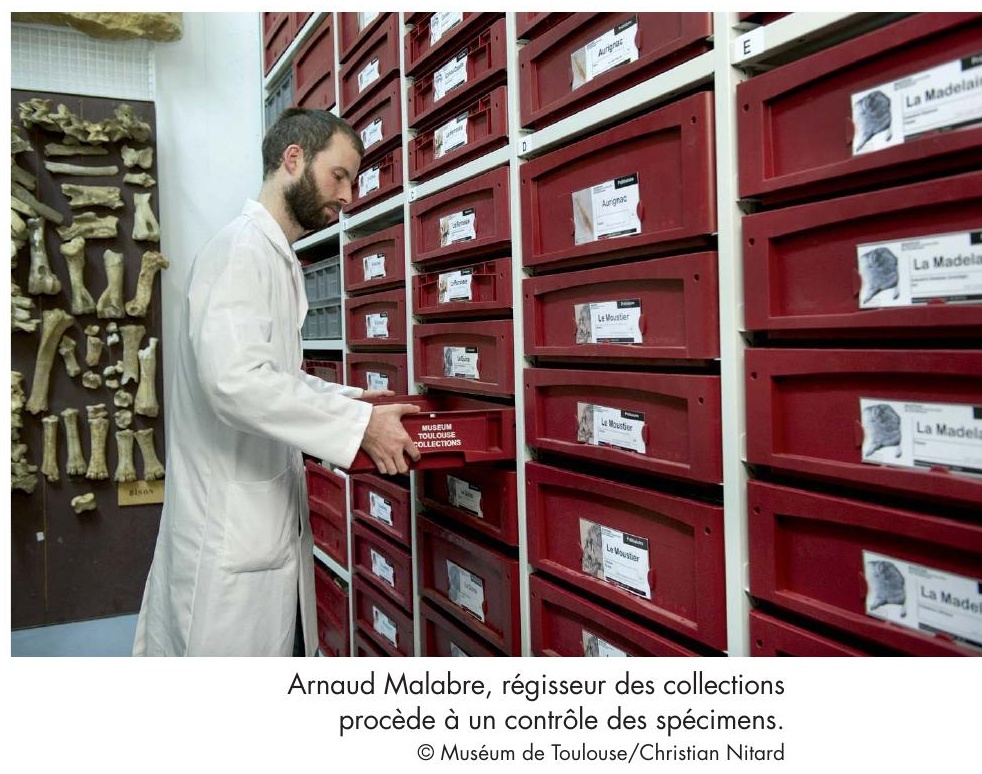

bases de données tant sur le plan de la gestion que sur celui de la documentation des collections. Ce n'est donc qu'à ce moment que la problématique du récolement et de l'informatisation «massive » des collections fut engagée. Un administrateur prit en charge les relations avec notre fournisseur, l'établissement d'une charte de saisie ainsi que la coordination et la vérification des fiches. De même, nous adaptions nos numéros d'inventaire (pour les enregistrements rétrospectifs) aux préconisations afin d'éviter la possibilité de doublons, ce qui permettait de continuer à pouvoir travailler simultanément sur les différentes bases. La multiplication des utilisateurs sur les trois bases ainsi que la participation temporaire de stagiaires et « saisonniers » fit apparaître encore de nombreuses différences quant à la saisie des données.

Ceci se confirma lors de la fusion et de la juxtaposition des deux bases de sciences naturelles. Ce fut alors l'occasion d'homogénéiser un certain nombre de thésaurus (lieux, personnes, taxonomie, localisations...) ainsi que leurs hiérarchies respectives.

À l'heure actuelle nous fonctionnons encore avec deux bases de données, d'un même fournisseur, mais 


\section{Points de vue complémentaires}

présentant des spécificités propres. Les données administratives, notamment celles concernant le mode et la date d'entrée dans les collections du musée, sont souvent difficiles à documenter et ce n'est qu'au coup par coup que nous arrivons à préciser ces informations. En effet, trop peu d'archives ont été dépouillées et aucun vrai cahier d'inventaire n'existe. Nous trouvons par contre plusieurs catalogues de collections, des registres d'entrées et des cahiers de laboratoire. L'ensemble de l'historique du muséum n'est pas totalement documenté et souvent de manière irrégulière. C'est jusqu'à la veille de la Première Guerre mondiale que nous trouvons le plus d'archives, les collections étant toutefois documentées de manière plus ou moins précise suivant les disciplines. Par la suite et ce pendant deux décennies, ce n'est pratiquement que grâce au préparateur (Philippe Lacomme) qui nota parfois avec une précision extrême son activité que nous arrivons à documenter les collections. Enfin, Gaston Astre publia, à la fin des années 1940, l'histoire de l'établissement.
Le récolement décennal a permis une mise à jour des bases informatiques. Ces bases correspondent à notre inventaire documentaire «dynamique ». L'inventaire réglementaire «immuable » est un extrait de ces bases de données reprenant seulement les objets patrimoniaux ainsi que les informations correspondant aux 18 colonnes «traditionnelles ». Cette phase est actuellement en cours et doit permettre, d'ici la fin de l'année 2014, l'édition de notre premier véritable inventaire réglementaire. 